\title{
Modifying the Estimated Ground Height to Mitigate Error Effects on Bipedal Robot Walking
}

\author{
Daniel Wahrmann, Tilman Knopp, Robert Wittmann, Arne-Christoph Hildebrandt, Felix Sygulla, \\ Philipp Seiwald, Daniel Rixen and Thomas Buschmann*
}

\begin{abstract}
Classic biped walking controllers assume a perfectly flat, rigid surface on which the robot walks. While walking over unknown terrain, robots need to sense and estimate the ground location. Errors in this estimation result in an unexpected early or late ground contact of the swing foot. In this paper, we analyze how these errors affect walking stability. Based on simulation results, we propose a strategy that mitigates this effect. We show that if the ground height has an associated uncertainty, an overestimation of its value results in a more stable walk. This overestimation depends on both sensor data and the robot's dynamics. By using a reduced robot model, our strategy could be implemented into the realtime control to make the robot more robust against perception errors and irregular surfaces.
\end{abstract}

Keywords: Autonomous Robotics, Biped Robots, Locomotion, Stabilization

\section{INTRODUCTION}

One of the main motivations behind robotic biped locomotion research is the potential, compared to wheeled vehicles, to navigate over uneven terrain. However, the high number of degrees of freedom $(D O F s)$ and underactuated characteristics make walking control considerably difficult and computationally expensive. Since its introduction in 1972 [1], the Zero Moment Point (ZMP) has been widely used as a starting point for walking motion generation with reduced robot models [2]-[6]. These representations, such as the Linear Inverted Pendulum Model (LIPM) [2] or a three point mass model [5], simplify the motion generation algorithms to such an extent that they can be performed in real-time. Feedback control is introduced to compensate for modeling errors [7][10]. These strategies can be easily combined with a vision system to navigate obstacle-free, flat regions [11]. Most are based on a vision system that can recognize walkable surfaces in the environment; these are sent to the walking control system, which adapts footstep locations and robot trajectories to navigate over these surface models [12]-[23]. However, a perfect ground location detection is assumed. These perception systems employ different kinds of on-board or external sensors that are subject to errors, regardless of the environment representation used afterwards. Gutmann et al. [12], [15] used a segmented 2.5D map to recognize surfaces above the ground with an error below $1.5 \mathrm{~cm}$. A similar strategy was used by Michel, Chestnutt, Nishiwaki et al. [14], [16], [18], who used a full-sized biped robot to walk over complex scenarios; reported perception errors

\footnotetext{
* Technical University of Munich, Chair of Applied Mechanics, Germany daniel.wahrmannetum. de
}

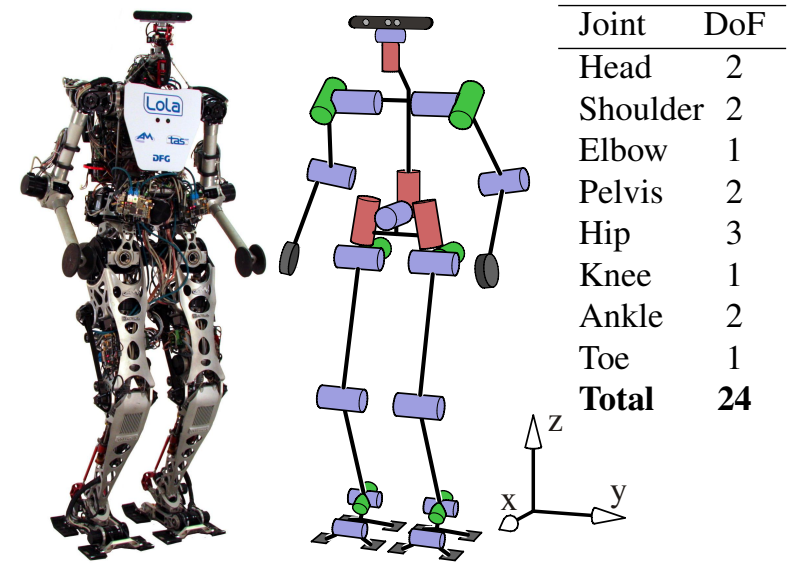

Fig. 1. Photo and kinematic structure of the humanoid robot Lola with an RGB-D sensor mounted on top.

lay around $1 \mathrm{~cm}$. Motivated by the DARPA Challenge [24], Stumpf et al. [19] and Fallon et al. [20] presented vision systems for the Atlas robot, with a ground detection error below $3 \mathrm{~cm}$.

In all works mentioned above, experiments were performed on static environments with relatively flat walking surfaces and at considerably slow walking speeds. Perception errors could be compensated by the robot's feedback stabilization system. When walking over more cluttered terrain and at higher speeds, biped robots may experience larger perception errors with a more pronounced effect on their stability [25]. In order to cope with such Early Contact (EC) or Late Contact $(L C)$ events, many authors exploit additional sensor information (typically, contact sensors in the robot's feet) and phase-switching mechanisms [25]-[29]. Additional robustness may be achieved with state estimation algorithms and real-time footstep modifications [30]-[32].

In summary, all strategies presented compensate perception errors with robust control. In this paper, in contrast, we consider modifying the environment representation, specifically the detected ground height. The premise is simple: "is it possible to find an optimal value for the ground height in terms of stability?". This question can become even more relevant when walking over irregular surfaces (e.g. gravel, grass), where a perception system could provide a variable accuracy factor (e.g. the standard deviation) for each detected surface. Additionally, for high walking speeds, vision 
systems are less precis $\mathrm{Q}^{1}$ due to motion blur and reduced filtering possibilities. For our work, we use a simplified model of our robot Lola (see Fig. 1). After outlining the scope of our work (Section III), we present our three-mass robot model (Section III). In Section IV, we analyze how $E C$ and $L C$ events affect the robot's stability and discuss different quantifiable stability indicators. In Section $\mathrm{V}$, we present a strategy for finding an optimal value for the ground height. As it depends on online parameters such as sensor information or the robot's dynamics, we propose using a reduced robot model that can be processed in real-time. Limitations and future work are discussed in Section VI Our analysis is based on the standard walking control of the robot Lola and validated using our full, multi-body simulation [33]. Nevertheless, our results can be easily applied to other biped robots.

\section{Problem Statement}

The analysis presented in this paper is not based on specific sensor data. The results we present rely only on the robot's dynamics and the consequences of $E C$ and $L C$ events. Therefore, it may be applicable to many different systems. However, our scenario consists of a biped robot and a perception system for ground (or surface) detection.

We assume that the error distribution $p$ of the ground estimation is known beforehand. For our work, we consider only the error in the vertical $(z)$ direction. In order to obtain computable results (Section V), we either assume the error to be constrained between finite limits or neglect errors outside those bonds. In general:

$$
1=\int_{-\infty}^{+\infty} p(z) d z \simeq \int_{z_{\min }}^{z_{\max }} p(z) d z
$$

with the limits $z_{\min }<z_{\max }$.

If we consider the system's expected ground height $z_{\exp } \in$ $\left[z_{\min }, z_{\max }\right]$ and the ground's real height $z_{\text {real }} \in\left[z_{\min }, z_{\max }\right]$, we can define the $E C, L C$ and Ideal Contact (IC) events:

$$
\begin{cases}E C & : z_{\text {exp }}<z_{\text {real }} \\ L C & : z_{\text {exp }}>z_{\text {real }} \\ I C & : z_{\text {exp }}=z_{\text {real }}\end{cases}
$$

In usual cases, $z_{\exp }$ is defined as the sensor's output or expected value $z_{\text {exp,sensor }}=E(Z)=\int_{-\infty}^{+\infty} z p(z) d z$. The objective of this work is to find an optimal value $z_{\text {exp,opt }}$ such that the effect of all possible $E C$ and $L C$ events on the robot's stability is minimized. In order to be applicable, the solution has to be computable online while the robot is walking.

To reduce computational costs and simplify our analysis, we restrict ourselves to the following case:

- The robot's control uses a fixed step duration $T_{\text {step }}$.

- We assume fixed phase durations and no footstep modification mechanisms since they are supposed to work in a later stage.

${ }^{1}$ In the following video, our robot Lola walks over an unexpected platform; the point cloud of the RGB-D sensor is noisier than usual: https://youtu.be/rKsx8HKvBkg
- The ground is horizontal and $z_{\text {exp,sensor }}=0$.

- Only the dynamics in the sagittal plane are considered. Classical concepts of stability are difficult to apply in nonlinear, non-smooth systems such as biped robots [33]. To the authors' knowledge, there is no clear practical definition and it is unclear how it could be quantified. In Section IV], we introduce different variables related to the robot's stability and analyze their potential applicability as quantifiable stability indicators to the present problem. In this work, we define a stability indicator as a function of the robot's state that is convex with respect to $\left(z_{\text {real }}-z_{\exp }\right)$ and presents a global minimum at $\left(z_{\text {real }}-z_{\text {exp }}\right)=0$.

\section{Model And Simulation}

As stated before, our application platform is the biped robot Lola. The reaction of the robot to unexpected ground heights could be evaluated in experiments, but other model errors and disturbances make it difficult to isolate a single factor. Repeatability can only be achieved in simulation. Therefore, we use as a reference our full multi-body simulation [33], which handles unilateral and compliant contacts and takes motor dynamics and control loops into consideration.

However, its high computational cost makes this simulation impractical for generating large amounts of data or implementing it in a real-time scenario (ref. Section $\mathrm{V}$ ). Therefore, we use an extension of the simplified three-mass model presented in [31] which can handle different ground heights. In the following, we explain the model's main properties.

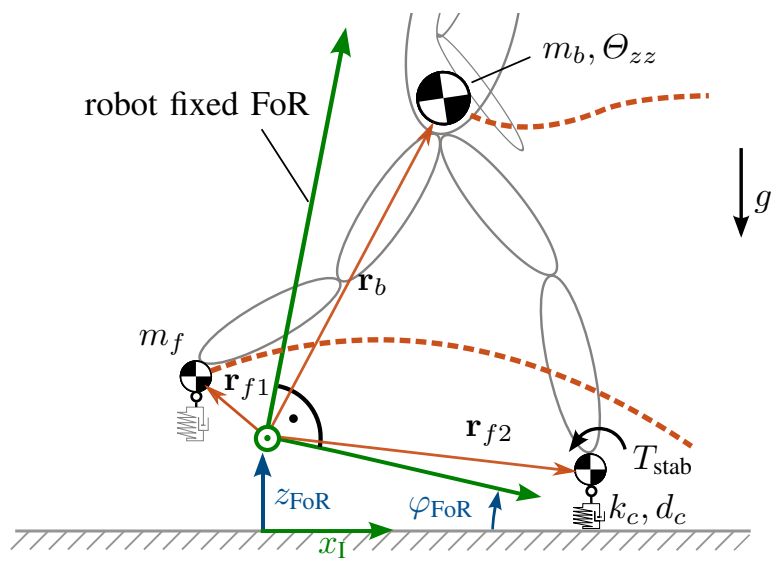

Fig. 2. Three-mass model in the saggital plane with two unactuated DoFs and unilateral compliant contacts.

Note that inverted pendulum models (such as the LIPM) become imprecise when the legs' dynamics are not negligible [30]. The robot is discretized then with one point mass for each leg $m_{f}$ at the feet and an upper body mith mass $m_{b}$ (near the center of mass) and inertia $\Theta_{z z}$ (ref. Fig. 2).

The upper body and feet are assumed to follow the ideal trajectories $\mathbf{r}_{b}$ and $\mathbf{r}_{f 1,2}$ in the robot's Frame of Reference (FoR). These are obtained from the simulated robot control. The underactuated state is simulated via two passive DOFs between the FoR and the ground $\left(x_{\mathrm{I}}-z_{\mathrm{I}}\right)$ : a vertical displacement $z_{\mathrm{FoR}}$ and an inclination on the sagittal plane $\varphi_{\mathrm{FoR}}$. 
Contact interactions between feet and ground are modeled as point vertical forces, with a unilateral, linear spring $\left(k_{c}\right)$ and damper $\left(d_{c}\right)$ model. The center of pressure is fixed to the contact point. The force control and upper body stabilization is taken into account with an additional stabilization variable $T_{\text {stab. }}$. It follows a PD-control (control gains $K_{p}$ and $K_{d}$ ) based on the one implemented in Lola's control:

$$
T_{\mathrm{stab}}=\operatorname{sat}\left(-K_{p} \varphi_{\mathrm{FoR}}-K_{d} \dot{\varphi}_{\mathrm{FoR}}\right)
$$

and is saturated at $\pm \frac{\text { sole length }}{2}\left(m_{b}+2 m_{f}\right) g$. A detailed description of the model's dynamics can be found in [31].

\section{ANALysis}

Using the model from Section III we simulate $E C, I C$ and $L C$ events by changing the ground height after achieving periodic motion. In Figs. 3 and 4 , a simulation example of an $E C$ and an $L C$ event, respectively, is shown. A different ground is defined for each foot to avoid lateral impacts and evaluate only changes in the $z$ direction. The forces on each foot and the inclination of the sagittal plane are shown, as well as the position of the robot (depicted with three segments: one for the upper body and two between $r_{b}$ and $\left.r_{f 1,2}\right)$ at three time instances $a, b$ and $c$ for reference.
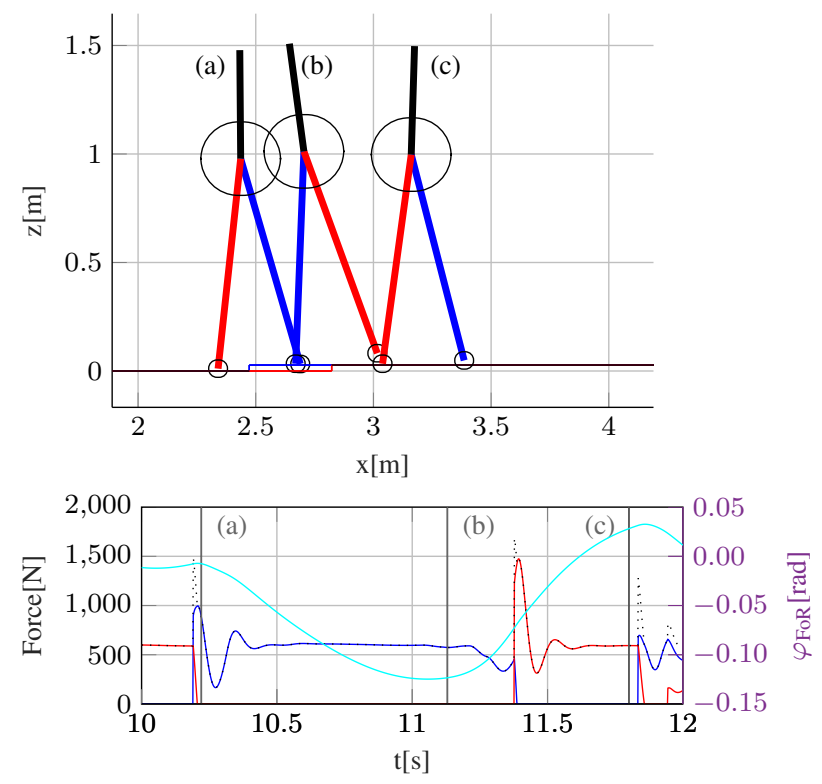

Fig. 3. A simulation of the three-mass model. Top: the robot position at 3 instances. Bottom: the progress of the contact forces for each foot (blue and red), total force (dashed) and the inclination of the sagittal plane (cyan) can be seen for an $E C$ event.

These simulations are repeated for different ground heights (positive for $E C$ and negative for $L C$ events). Thus, the value of different indicators can be evaluated against the $I C$ case. Additionally, this is performed for different walking parameters. As an example, Fig. 5 shows the plot of the angular momentum with respect to the origin for one set of walking parameters and $z_{\text {real }}$ varying between $-0.05 \mathrm{~m}$ and $0.03 \mathrm{~m}$. As the foot's trajectory has a maximum height $h$ of $0.03 \mathrm{~m}$, higher values of $z_{\text {real }}$ are not relevant. Besides, the
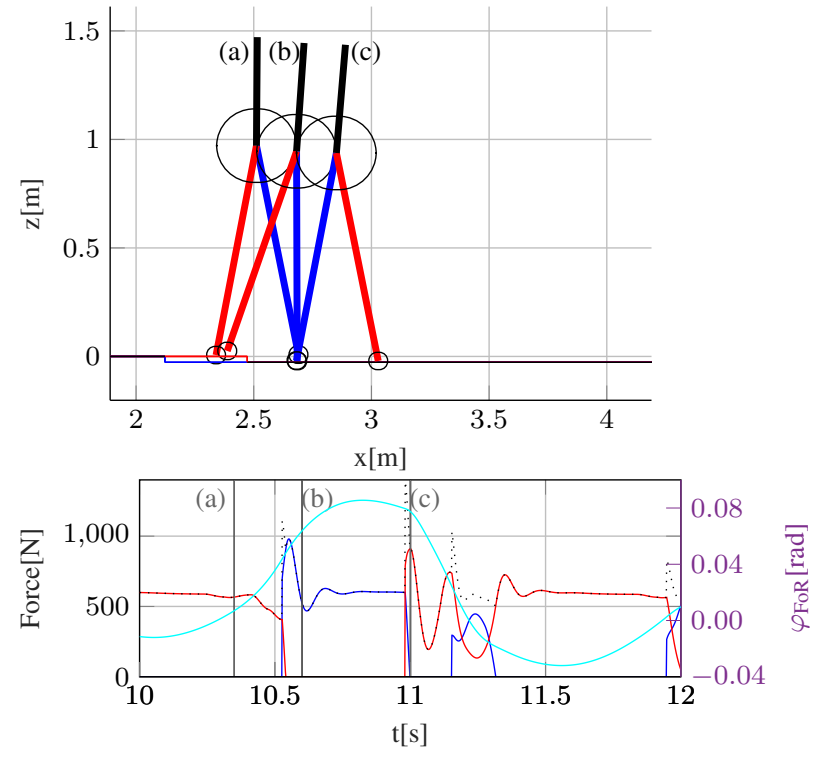

Fig. 4. A simulation of the three-mass model. Top: the robot position at 3 instances. Bottom: the progress of the contact forces for each foot (blue and red), total force (dashed) and the inclination of the sagittal plane (cyan) can be seen for an $L C$ event.

TABLE I

Simulation Parameters

\begin{tabular}{c|c} 
Walking step duration $T$ & $0.8 \mathrm{~s}$ \\
\hline$h$ & $0.03 \mathrm{~m}$ \\
\hline Time discretization & $0.0015 \mathrm{~s}$ \\
\hline Saturation of $T_{\text {stab }}$ & $\pm 80 \mathrm{Nm}$ \\
\hline Walking step length $s$ & $0.25 \mathrm{~m} 0.3 \mathrm{~m} 0.35 \mathrm{~m} 0.4 \mathrm{~m} 0.45 \mathrm{~m}$ \\
\hline$z_{\text {real }}$ & between $-0.05 \mathrm{~m}$ and $0.03 \mathrm{~m}$ \\
\hline Simulation duration & $4.8 \mathrm{~s}$
\end{tabular}

simulations result in the robot loosing balance before reaching those limit values. In the following, we discuss some of the stability indicators considered. Due to the high number of parameters, it is not possible to include all simulation results in this paper. Instead, a small selection based on the parameters provided in Table 1 will be presented.

\section{A. Indicators}

The sagittal inclination of the FoR $\varphi_{\mathrm{FoR}}$ (upper body inclination) is a potential indicator: a high absolute value means that the robot is falling. Nevertheless, during normal walking, it oscillates between $-0,012 \mathrm{rad}$ and $+0,011 \mathrm{rad}$. Its periodic motion is not easy to compare or quantify, so we consider the minimum and maximum values $\varphi_{\min }, \varphi_{\max }$ along one run. In the case of an $E C$, the robot tilts backwards and $\varphi_{\min }$ decreases while $\varphi_{\max }$ stays relatively constant. On the other hand, the robot tilts forward in the case of an $L C$ and $\varphi_{\max }$ increases while $\varphi_{\min }$ stays relatively constant (ref. Fig. 9). This behavior is strongly dependent on $z_{\text {real }}$ but not on the walking parameters, except for large values of $\left|z_{\text {real }}\right|$. An indicator considering both effects (e.g. $\left.\max \left(\left|\varphi_{\mathrm{FoR}}\right|\right)\right)$ can be applied to our problem.

Note that the robot's stability is not only influenced by the first contact with an unexpected floor height: if we assume no 
more changes on the floor height, the step after an $E C$ event consists of an $L C$, and vice-versa; this is due to the change in the robot's state, especially how $\varphi_{\mathrm{FoR}}$ is affected by $E C$ and $L C$ events (see Figs. 3 and 4 ). Thus we also observe the values of $\varphi_{\text {FoR }}$ at the first and second contact with the ground after the change in $z_{\text {real }}, \varphi_{\mathrm{con}_{1}}$ and $\varphi_{\mathrm{con}_{2}} \cdot \varphi_{\mathrm{con}_{1}}$ shows quasilinear behaviors for small values of $z_{\text {real }}$. More interesting is the behavior of $\varphi_{\mathrm{con}_{2}}$ which is almost 0 for a range of low values of $\left|z_{\text {real }}\right|$ and shows a sudden increase of absolute value outside of that region. This can be explained by the effect of $T_{\text {stab }}$, which is able to counteract small variations of $z_{\text {real }}$. Note that $\varphi_{\mathrm{con}_{2}}$ indicates the state of the robot at the contact with the ground, where a sudden change in the acting force will hinder the stabilization controller. An indicator such as $\left|\varphi_{\mathrm{con}_{2}}\right|$ is therefore meaningful for our problem.

The angular momentum $L^{0}$ with respect to the inertial coordinate system $\left(x_{\mathrm{I}}-z_{\mathrm{FoR}}\right.$ in Fig. 2) also indicates future states of instability. Fig. 5 shows the value of $L^{0}$ for one set of walking parameters and different values of $z_{\text {real }}$. As

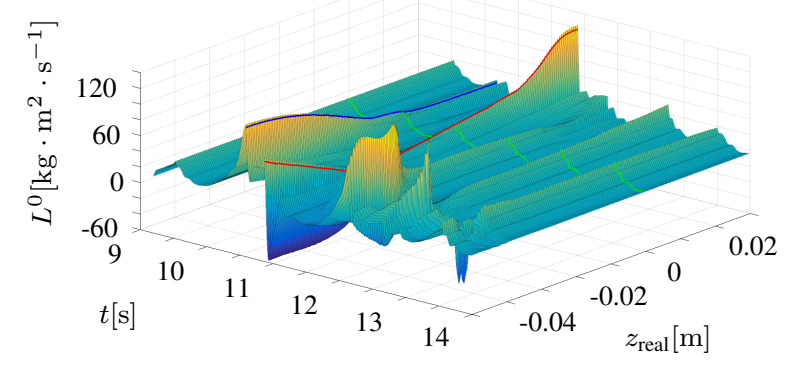

Fig. 5. Angular momentum with respect to the inertial coordinate system for a walking step length of $s=0.35 \mathrm{~m}$. The $I C$ simulation is depicted in green while the values at the time of the first and second contacts with the modified ground are highlighted in blue and red, respectively.

with $\varphi_{\mathrm{FoR}}$, we plot the maximum and minimum values of $L^{0}, L_{\min }^{0}$ and $L_{\max }^{0}$. These are shown for different walking parameters in Fig. 6, which can be interpreted as the limits of the projection of Fig. 5 on the $z_{\text {real }}-L^{0}$ plane. Even though

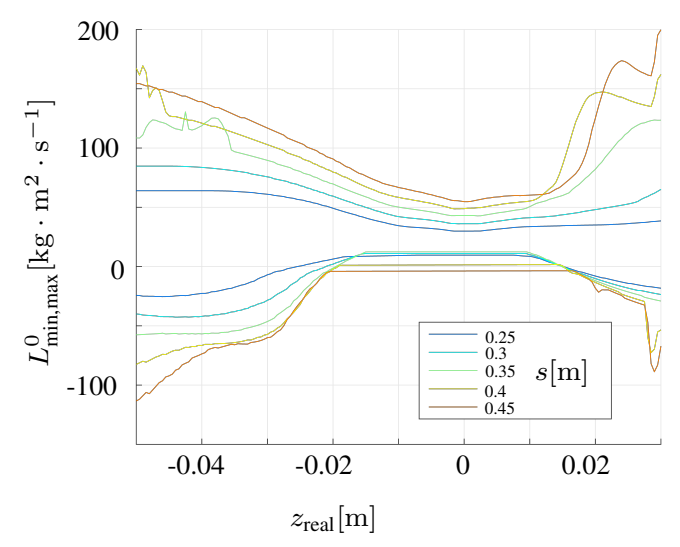

Fig. 6. Maximum and minimum values of $L^{0}$ along $z_{\text {real }}$ for different simulations.

no discernible trend can be found on $L_{\max }^{0}, L_{\min }^{0}$ shows a clear constant value for a range around $z_{\text {real }}=0$ and an increasing deviation outside of it. This behavior allows for a convex indicator in the form of e.g. $\left(L_{\min }^{0}(0)-L_{\min }^{0}\left(z_{\text {real }}\right)\right)$. The angular momentum with respect to the FoR $L^{\mathrm{FoR}}$ has been proposed as a stability indicator in [34], but was already dismissed by [33] for the robot Lola. Our simulations showed no clear trend (no convexity) as well. Other indicators analyzed were the distance of the ZMP to the foot's edge, the value of $T_{\text {stab }}$ and its minima and maxima. However, none of them show a near convex/concave behavior with respect to $\left(z_{\text {real }}-z_{\text {exp }}\right)$ and are not applicable to our problem.

Nevertheless, if we consider the timespan $t_{\text {sat }}$ in which $T_{\text {stab }}$ is saturated, we obtain another indicator, as any values above zero indicate a limitation of the stability control and thus a potential state of instability (see Fig. 7).

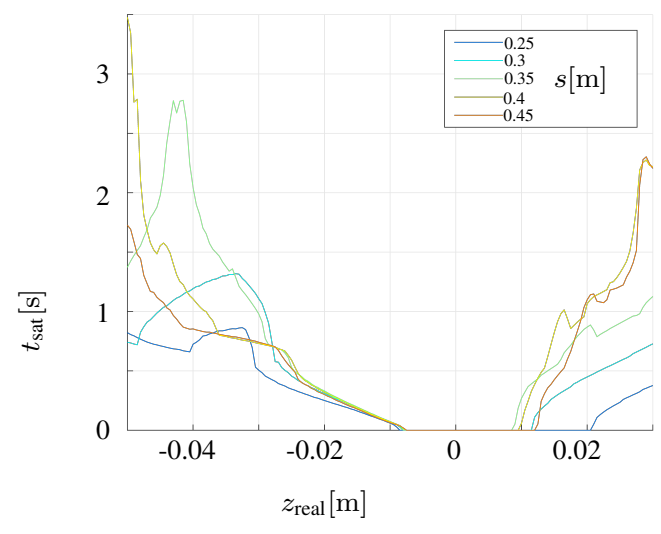

Fig. 7. Timespan in which $T_{\text {stab }}$ is saturated along $z_{\text {real }}$ for different simulations.

\section{B. Discussion}

The results presented here are strongly dependent on Lola's particular walking controller and model parameters. Nevertheless, the presented strategy and indicators can be easily applied to other robots. Of all variables analyzed, several fulfill our premise and can be applied to our problem (namely, $\varphi_{\max , \min }, \varphi_{\mathrm{con}_{2}}, L_{\min }^{0}, t_{\mathrm{sat}}$ ). The indicators can be seen in Fig. 8 for one set of walking parameters. They are all related to stability in the sense that they can indicate states in which the disturbance is too large to be compensated by the stabilization torque. There is a priori no criteria by which some may be better than others for our problem. Those that are less dependent on the walking parameters could potentially simplify the implementation (see Section V.

\section{Model Validation}

As shown in previous work, the dynamics of a robot such as Lola can be well described by our three-mass model; it allows one to reliably predict future states of instability and reactions to disturbances [30]-[32]. In order to confirm the validity of the results, we performed full, multi-body simulations for different ground heights. In Fig. 9, it can be seen that they show a similar trend and are almost proportional, which does not affect the validity of our solution. 

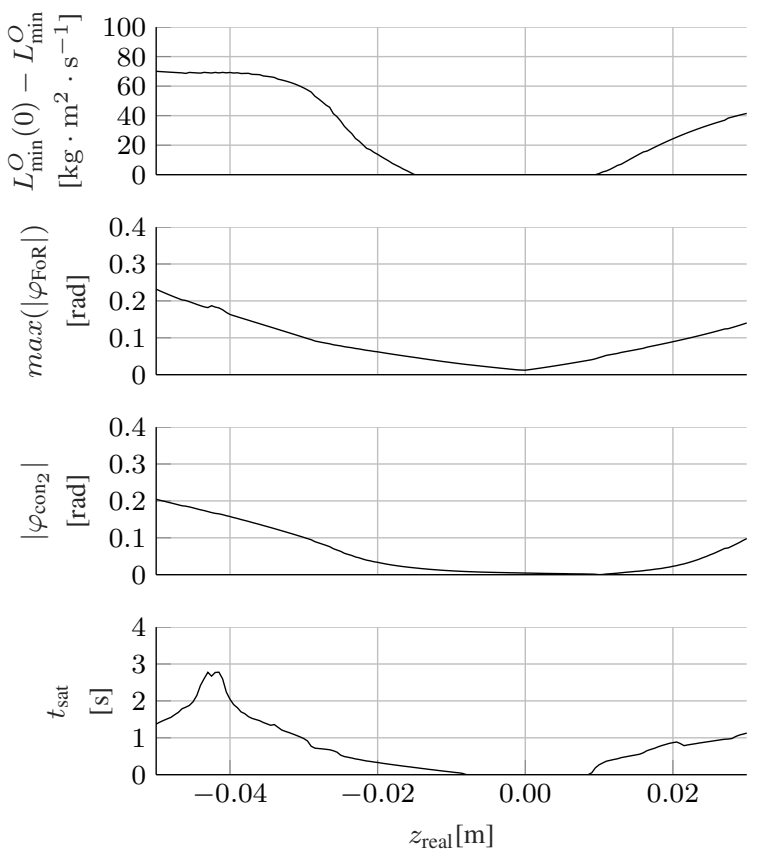

Fig. 8. A comparison of selected stability indicators for $s=0.35 \mathrm{~m}$.

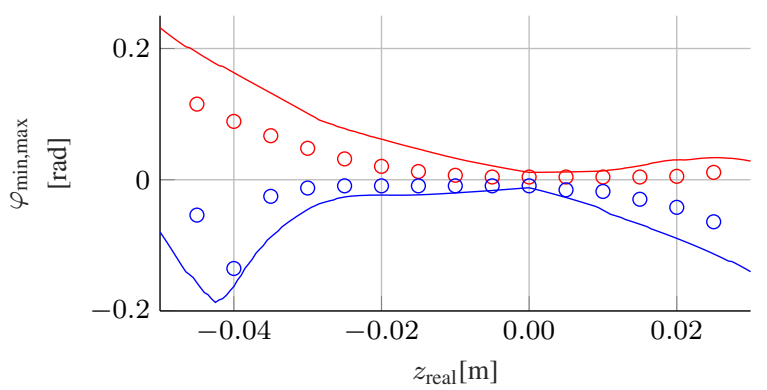

Fig. 9. Validation of results for $\varphi_{\max }$ (red) and $\varphi_{\min }$ (blue) with $s=$ $0.35 \mathrm{~m}$. Lines: simple model. Dots: full multi-body simulation.

\section{Application in Walking Controller}

\section{A. Solution}

As explained in Section III the objective of this work is to find the optimal expected ground height $z_{\text {exp,opt }}$ according to a stability indicator. Naturally, a weighted combination of several of the presented indicators can be used as an indicator. Let $z_{\max }-z_{\min }=l$. For our solution, we take an indicator $K$ defined in $[-l, l]$, with $z_{\exp }=0$ and such that a smaller value of $K$ can be associated with a more stable walk.

Considering any possible value of $z_{\exp }$, we define $\tilde{z}=$ $z_{\text {real }}-z_{\text {exp }}$. Thus, we can calculate the indicator value as:

$$
K_{z_{\exp }}=K\left(z_{\text {real }}-z_{\exp }\right)=K(\tilde{z})
$$

We propose then, as an optimal expected value, the one for which the accumulated effect of all possible $E C, L C$ and $I C$ events is minimal:

$$
z_{\text {exp,opt }}=\underset{\hat{z} \in\left[z_{\min }, z_{\max }\right]}{\arg \min } \int_{z_{\min }}^{z_{\max }} p(z) K(z-\hat{z}) d z
$$

Let us assume that $p$ is symmetric with respect to $z_{\text {exp,sensor }}$. For a symmetric indicator $K$, we obtain $z_{\text {exp,opt }}=z_{\text {exp,sensor }}$. In that case, the influence of $E C$ and $L C$ events would be equivalent. It is interesting, however, that after solving $z_{\text {exp,opt }}$ for several simulation scenarios with the indicators presented in this paper, we obtain $z_{\text {exp,opt }}>z_{\text {exp,sensor }}$. This result, which is consequent with the asymmetry of Fig. 8, indicates that EC events have a greater effect on stability than LC events, and $z_{\exp }$ should be overestimated. The precise value (5) depends both on the sensor's error and robot's dynamics and cannot be computed beforehand.

\section{B. Implementation}

The presented solution is very general so that it can be applied regardless of the model used for analysis. Nevertheless, it is computationally expensive. A thorough implementation would involve the following process:

a) perform $E C, L C$ and $I C$ event simulations for a full robot model in the range $\tilde{z} \in[-l, l]$, and obtain the values for $K(\tilde{z})$

b) with the result from a), calculate the integral term in 5 for the range $z_{\exp } \in\left[z_{\min }, z_{\max }\right]$ and find the minimum

that would have to be performed every step, as a depends on the sensor's input and walking parameters. Out of both operations, b is computationally inexpensive while a) presents the most difficulty.

In order to implement a in the real-time walking controller, an initial solution could be to precompute it offline. Out of a database with detailed simulation results, the values of $K(\tilde{z})$ could be interpolated during walking. One drawback of this method is that it makes a change in the stability indicator difficult. Besides, the amount of variables involved would result in a large amount of data. On the other hand, if the discretization is coarse, then the result's precision would decrease. Furthermore, it is not clear if an interpolation could work in this high-dimensional problem.

We propose using a reduced robot model (see Section III) to perform a large number of simulations online and obtain values for $K(\tilde{z})$. As shown in previous work [31], simulations of such a reduced model are considerably efficient and can be implemented into the real-time control. Besides, as explained before, such a reduced model can still reliably predict the robot's dynamics. If we consider that a small modification of the ground height does not necessarily imply a re-planning of the footstep positions, these simulations may be performed throughout one walking step, which is enough for obtaining acceptable results. We plan to implement this solution in our walking controller in order to further evaluate the stability indicators and validate the applicability of our model.

\section{CONCLUSiOnS}

In this paper, we presented an analysis of the effect of perception errors on the stability of biped robots. Based on a simplified robot model, several stability indicators were suggested. We propose the application of this model to find an optimal value for the assumed ground height in 
the presence of bounded uncertainty. This could potentially improve the robustness of the robot against perception errors, without making changes to the walking control. An accuracy factor describing irregular surfaces (e.g. gravel, grass) can also be taken into account. According to our results, an overestimation of the ground height leads to a more stable walk in most cases.

Some limitations of this work include the reduced model used for analysis and the strong assumptions for the test scenario. We intend to evaluate the dynamics in the lateral as well as the sagittal plane and apply them in a combined solution. For the future, we plan to implement the proposed strategy on the robot Lola and validate the results presented in this paper.

\section{ACKNOWLEDGMENT}

This work is supported by the DAAD and the DFG (project BU 2736/1-1).

\section{REFERENCES}

[1] M. Vukobratovic and J. Stepanenko, "On The Stability of Anthropomorphic Systems," Mathematical Bioscience, vol. 15, pp. 1-37, 1972.

[2] S. Kajita and K. Tani, "Study of Dynamic Biped Locomotion on Rugged Terrain - Derivation and Application of the Linear Inverted Pendulum Mode," in IEEE International Conference on Robotics and Automation, 1991.

[3] K. Loffler, M. Gienger, and F. Pfeiffer, "Model Based Control of a Biped Robot," in 7th IEEE International Workshop on Advanced Motion Control, 2002.

[4] K. Nishiwaki and S. Kagami, "High Frequency Walking Pattern Generation based on Preview Control of ZMP," in IEEE-RAS International Conference on Humanoid Robots, 2006.

[5] T. Buschmann, S. Lohmeier, M. Bachmayer, H. Ulbrich, and F. Pfeiffer, "A Collocation Method for Real-Time Walking Pattern Generation," in IEEE-RAS International Conference on Humanoid Robots, 2007.

[6] T. Takenaka, T. Matsumoto, and T. Yoshiike, "Real Time Motion Generation and Control for Biped Robot - 1st Report: Walking Gait Pattern Generation-," in IEEE/RSJ International Conference on Intelligent Robots and Systems, 2009, pp. 1084-1091.

[7] S. Kajita, T. Nagasaki, K. Kaneko, K. Yokoi, and K. Tanie, "A Running Controller of Humanoid Biped HRP-2LR," in IEEE International Conference on Robotics and Automation, 2005.

[8] T. Buschmann, S. Lohmeier, and H. Ulbrich, "Biped Walking Control Based on Hybrid Position/Force Control," in IEEE/RSJ International Conference on Intelligent Robots and Systems, 2009.

[9] T. Takenaka, T. Matsumoto, T. Yoshiike, T. Hasegawa, S. Shirokura, H. Kaneko, and A. Orita, "Real Time Motion Generation and Control for Biped Robot -4th report: Integrated Balance Control-," in IEEE/RSJ International Conference on Intelligent Robots and Systems, 2009.

[10] K. Nishiwaki and S. Kagami, "Strategies for Adjusting the ZMP Reference Trajectory for Maintaining Balance in Humanoid Walking," in IEEE International Conference on Robotics and Automation, 2010.

[11] T. Buschmann, S. Lohmeier, M. Schwienbacher, V. Favot, H. Ulbrich, F. Von Hundelshausen, G. Rohe, and H.-J. J. Wuensche, "Walking in Unknown Environments - a Step Towards More Autonomy," in IEEE-RAS International Conference on Humanoid Robots, 2010.

[12] J. Gutmann, M. Fukuchi, and M. Fujita, "A Floor and Obstacle Height Map for 3D Navigation of a Humanoid Robot," in IEEE International Conference on Robotics and Automation, 2005.

[13] R. Cupec and G. Schmidt, "An Approach to Environment Modelling for Biped Walking Robots," in IEEE/RSJ International Conference on Intelligent Robots and Systems, 2005.

[14] P. Michel, J. Chestnutt, S. Kagami, K. Nishiwaki, J. Kuffner, and T. Kanade, "GPU-accelerated Real-Time 3D Tracking for Humanoid Locomotion and Stair Climbing," in IEEE International Conference on Intelligent Robots and Systems, 2007.
[15] J. S. Gutmann, M. Fukuchi, and M. Fujita, "3D Perception and Environment Map Generation for Humanoid Robot Navigation," The International Journal of Robotics Research, vol. 27, no. 10, 2008.

[16] J. Chestnutt, Y. Takaoka, K. Suga, K. Nishiwaki, J. Kuffner, and S. Kagami, "Biped Navigation in Rough Environments using On-board Sensing," in IEEE/RSJ International Conference on Intelligent Robots and Systems, 2009.

[17] S. Oßwald, J. S. Gutmann, A. Hornung, and M. Bennewitz, "From 3D Point Clouds to Climbing Stairs: A Comparison of Plane Segmentation Approaches for Humanoids," in IEEE-RAS International Conference on Humanoid Robots, 2011.

[18] K. Nishiwaki, J. Chestnutt, and S. Kagami, "Autonomous Navigation of a Humanoid Robot over Unknown Rough Terrain using a Laser Range Sensor," The International Journal of Robotics Research, vol. 31, no. 11, pp. 1251-1262, 2012.

[19] A. Stumpf, S. Kohlbrecher, D. C. Conner, O. von Stryk, O. V. Stryk, and O. von Stryk, "Supervised Footstep Planning for Humanoid Robots in Rough Terrain Tasks using a Black Box Walking Controller," in IEEE-RAS International Conference on Humanoid Robots, 2014.

[20] M. F. Fallon, P. Marion, R. Deits, T. Whelan, M. Antone, J. Mcdonald, and R. Tedrake, "Continuous Humanoid Locomotion over Uneven Terrain using Stereo Fusion," in IEEE-RAS International Conference on Humanoid Robots, 2015.

[21] A.-C. Hildebrandt, D. Wahrmann, R. Wittmann, D. Rixen, and T. Buschmann, "Real-Time Pattern Generation Among Obstacles for Biped Robots," in IEEE/RSJ International Conference on Intelligent Robots and Systems, 2015.

[22] D. Wahrmann, A.-C. Hildebrandt, R. Wittmann, F. Sygulla, D. Rixen, and T. Buschmann, "Fast Object Approximation for Real-Time 3D Obstacle Avoidance with Biped Robots," in IEEE International Conference on Advanced Intelligent Mechatronics., 2016.

[23] A.-C. Hildebrandt, M. Demmeler, R. Wittmann, D. Wahrmann, F. Sygulla, D. Rixen, and T. Buschmann, "Real-Time Predictive Kinematic Evaluation and Optimization for Biped Robots," in IEEE/RSJ International Conference on Intelligent Robots and Systems, 2016.

[24] "DARPA Robotics Challenge (DRC)," 2017. [Online]. Available: http://archive.darpa.mil/roboticschallenge/

[25] T. Buschmann, A. Ewald, H. Ulbrich, and A. Buschges, "Event-Based Walking Control - From Neurobiology to Biped Robots," in IEEE/RSJ International Conference on Intelligent Robots and Systems, 2012.

[26] J. E. Pratt and G. A. Pratt, "Exploiting Natural Dynamics in the Control of a Planar Bipedal Walking Robot," in 36th Annual Allerton Conference on Communication, Control, and Computing, 1998.

[27] J. Pratt, C.-M. Chew, A. Torres, P. Dilworth, and G. Pratt, "Virtual Model Control: An Intuitive Approach for Bipedal Locomotion," The International Journal of Robotics Research, vol. 20, no. 2, pp. 129143, 2001.

[28] K. Sreenath, H.-W. Park, I. Poulakakis, and J. W. Grizzle, "A Compliant Hybrid Zero Dynamics Controller for Stable, Efficient and Fast Bipedal Walking on MABEL," The International Journal of Robotics Research, vol. 30, no. 9, pp. 1170-1193, 2011.

[29] M. Morisawa, F. Kanehiro, K. Kaneko, S. Kajita, and K. Yokoi, "Reactive Biped Walking Control for a Collision of a Swinging Foot on Uneven Terrain," in IEEE-RAS International Conference on Humanoid Robotics, 2011.

[30] R. Wittmann, A.-C. Hildebrandt, D. Wahrmann, D. Rixen, and T. Buschmann, "State Estimation for Biped Robots Using Multibody Dynamics," in IEEE/RSJ International Conference on Intelligent Robots and Systems, 2015, pp. 2166-2172.

[31] _ _ "Real-Time Nonlinear Model Predictive Footstep Optimization for Biped Robots," in IEEE-RAS International Conference on Humanoid Robots, 2015.

[32] R. Wittmann, A.-C. Hildebrandt, D. Wahrmann, F. Sygulla, D. Rixen, and T. Buschmann, "Model-Based Predictive Bipedal Walking Stabilization," in IEEE-RAS International Conference on Humanoid Robots, 2016.

[33] T. Buschmann, "Simulation and Control of Biped Walking Robots," Ph.D. dissertation, Technische Universität München, 2010.

[34] A. Goswami and V. Kallem, "Rate of change of angular momentum and balance maintenance of biped robots," in IEEE International Conference on Robotics and Automation, 2004. 